\title{
Limitación del esfuerzo terapéutico en la práctica clínica. Percepciones de profesionales médicos y de enfermería de unidades de pacientes críticos de un hospital público de adultos en la Región Metropolitana
}

\author{
María Cristina Paredes Escobar ${ }^{1}$
}

\begin{abstract}
Resumen: Uno de los problemas centrales de la bioética clínica relacionados con el final de la vida es la limitación del esfuerzo terapéutico. Su correcta práctica se traduce en limitar esfuerzos cuando las circunstancias del paciente dan la certeza de que no existen posibilidades de recuperación. El objetivo de este estudio fue conocer sobre este problema desde las percepciones de profesionales médicos y de enfermería que trabajan en unidades de pacientes críticos. Para ello se realizó un estudio cualitativo de tipo exploratorio y se aplicó teoría fundamentada como herramienta de análisis, a través de la búsqueda de categorías teóricas a partir de los datos. Entre los resultados globales se destaca que la limitación del esfuerzo terapéutico es una práctica habitual a la que se deben enfrentar los profesionales. Los conceptos técnicos se encuentran claros; sin embargo, los principales problemas éticos se originan en los procesos de toma de decisiones, ya que existe un desconocimiento de las implicancias éticas y una escasa reflexión sobre el tema. En ninguno de los dos grupos de estudio existían profesionales con formación en bioética, hecho frecuente en este ámbito.
\end{abstract}

Palabras clave: limitación del esfuerzo terapéutico, profesionales de salud, unidades de pacientes críticos, bioética clínica

\section{Limits to therapeutic efforts in clinical practice. Perceptions of medical and nursing professionals of critical patients units of a public hospital for adults in the Metropolitan Region}

\begin{abstract}
One of the main problems of clinical bioethics related to the end of life is the limits to therapeutic efforts. Its fair practice is reduced to limit efforts when the circumstances of the patient point to the lack of possibilities for recovering. The goal of this study is to inquire about this problem under the perceptions of medical and nursing professionals who work in critical patients units. An exploratory qualitative study was carried out applying based theory as tool for analysis, through the search of theoretical categories in data. Among the global results, the limits to therapeutic efforts are highlighted as a habitual practice which professionals must face. Technical concepts are found clear; nevertheless, the main ethical problems are originated in the processes of decision making, since there is lack of knowledge on the ethical implications and a scarce reflection about the topic. Both groups of study lacked professionals trained in bioethics, frequent fact in this field.
\end{abstract}

Key Words: limits to therapeutic efforts, health care professionals, critical patients units, clinical bioethics

\section{Limitaçáo do esforço terapêutico na prática clínica. Percepçóes de profissionais médicos e enfermeiros de unidades de pacientes críticos de um hospital público de adultos na Regiáo Metropolitana}

Resumo: Um dos problemas centrais da bioética clínica relacionados com o final da vida é a limitação do esforço terapêutico. A sua correta prática se traduz em limitar esforços quando as circunstâncias do paciente dão a certeza de que não existem possibilidades de recuperação. O objetivo deste estudo foi conhecer este problema a partir das percepçóes de profissionais médicos e enfermeiros que trabalham em unidades de pacientes críticos. Para isso se realizou um estudo qualitativo de tipo exploratório e se aplicou uma teoria fundamentada como ferramenta de análise através da busca de categorias teóricas a partir dos dados. Entre os resultados globais se destaca que a limitação do esforço terapêutico é uma prática habitual a qual os profissionais devem enfrentar. Os conceitos técnicos se encontram claros; entretanto, os principais problemas éticos se originam nos processos de tomada de decisóes, já que existe um desconhecimento das implicações éticas e uma escassa reflexão sobre o tema. Em nenhum dos dois grupos de estudo existiam profissionais com formação em bioética, fato frequente neste âmbito.

Palavras-chave: limitação do esforço terapêutico, profissionais de saúde, unidades de pacientes críticos, bioética clínica

\footnotetext{
${ }^{1}$ Licenciada en Enfermería, Magíster en Bioética. Profesora Escuela de Enfermería, Universidad de Chile, Chile Correspondencia: mariacristinaparedes@hotmail.es
} 


\section{Introducción}

Desde el nacimiento de las unidades de cuidados intensivos y las técnicas de soporte vital, el profesional de la salud se ha visto enfrentado a un abanico de posibilidades terapéuticas, que han ayudado a prolongar la vida y a restablecer la salud en relación con patologías que antes no tenían ninguna esperanza de recuperabilidad.

"Los cuidados intensivos nacieron cuando comenzó a ser posible la sustitución de funciones vitales, y por tanto el control de algunos de los procesos que solían conducir a la muerte: parada cardíaca, insuficiencia respiratoria, fracaso renal, etc. Fue en los años 60 cuando estas técnicas de soporte vital comenzaron a tener alguna relevancia sanitaria, y cuando el médico empezó a tener, por primera vez en la historia, un control efectivo sobre la muerte" (1).

Sin duda, la medicina intensiva planteó algunos de los principales problemas que dieron origen a la bioética y, por lo mismo, el enfoque ético de la clínica brinda la oportunidad de enriquecer y entregar herramientas para el proceso de toma de decisiones en el final de la vida.

Hoy la muerte ha sido medicalizada. Ocurre en los hospitales considerados como lugares fríos y deshumanizados; el médico históricamente ha considerado a la muerte como su peor enemiga $y$, por lo tanto, su desenlace como el fracaso de la praxis médica. En ocasiones, los profesionales no conocen los límites de las posibilidades terapéuticas, pero, sin lugar a dudas, el criterio fundamental de valoración debe ser la defensa y promoción del bien integral de la persona humana. Por consiguiente, cualquier intervención médica sobre la persona no solo está sometida a los límites de lo que es técnicamente posible, sino también determinada por el respeto a la misma naturaleza humana.

La limitación del esfuerzo terapéutico en las etapas finales de la vida se justifica cuando las circunstancias del paciente nos dan la certeza de que no existen posibilidades terapéuticas. "La limitación de esfuerzo terapéutico es la decisión meditada sobre la no implementación o la retirada de las terapéuticas médicas al anticipar que no conlle- varán un beneficio al paciente"(2). Esta debe ser aplicada a aquellos pacientes que, según el juicio médico, cursan un estado de irrecuperabilidad, ya sea porque son enfermos terminales, o bien, que sin serlos, a raíz del curso de su enfermedad, se ven cercanos a la muerte a pesar de la terapia aplicada.

El ingreso de los pacientes a las unidades críticas se justifica cuando existen claras posibilidades de beneficio para él. Pero, ¿qué sucede cuando esto no es posible? ¿Cuando, a pesar de los esfuerzos terapéuticos, el paciente se aproxima al proceso del morir y lo que queda es limitar esfuerzos y dar paso a completar el proceso del final de la vida? ¿Cómo enfrentan los profesionales esta situación? ¿Desde qué perspectiva y con qué valores?

El trabajo que ahora se informa tuvo como objetivo principal describir la práctica clínica de la limitación del esfuerzo terapéutico desde la perspectiva de profesionales médicos y de enfermería que trabajan en unidades de pacientes críticos. De esta forma, buscó descubrir esta realidad desde la propia mirada de los protagonistas, es decir, cómo viven ellos esta situación en su práctica diaria y cómo enfrentan estas experiencias. Con esta indagación se pretende contribuir al desarrollo de la cada vez más necesaria bioética en la práctica clínica.

\section{Métodos}

Se realizó un estudio de tipo cualitativo que utilizó como enfoque metodológico la teoría fundamentada de Strauss y Corbin (2002). "Se refieren a una teoría derivada de datos recopilados de manera sistemática y analizados por medio de un proceso de investigación. En este método, la recolección de datos, el análisis y la teoría que surgirá de ellos guardan estrecha relación entre sî"(3). Se buscó describir los procesos de sentido que dan lugar a la práctica clínica de la limitación del esfuerzo terapéutico desde la perspectiva del sujeto de investigación, indagando en profundidad la comprensión del fenómeno. Las proposiciones teóricas surgen luego de los datos obtenidos en la investigación, más que de los estudios previos.

Posteriormente, se utilizó el enfoque de ética empírica integrada. "Este enfoque supone la co- 
operación intensiva entre eticistas y científicos descriptivos, con la intención de integrar datos empíricos con teoría ética para alcanzar una conclusión normativa respecto a una práctica social específica" (4), y así vincular los datos elaborados en una teoría fundamentada con la reflexión bioética, para obtener las conclusiones de esta investigación.

La unidad de análisis estuvo compuesta por profesionales médicos y de enfermería de tres unidades de pacientes críticos de un hospital público de adultos. El universo se compone por un total de 49 sujetos: 17 médicos y 32 enfermeros. Se trabajó con una muestra del total de los profesionales mencionados que se obtuvo por saturación teórica de la información ${ }^{2}$.

Se aplicó un muestreo teórico, orientado al propósito de la obtención de declaraciones enriquecedoras que dieran cuenta de las dimensiones de la experiencia. Este se realizó persona a persona a través de una muestra intencionada, con el fin de aceptar los datos posibles de conseguir, lo que aportó al estudio resultados importantes de manera inesperada.

La recolección de los datos se realizó a través de una entrevista semiestructurada, aplicada y grabada previo consentimiento informado. Incluyó preguntas cerradas y abiertas. Las preguntas cerradas corresponden a la recolección de datos demográficos y de formación en bioética de los sujetos de estudio; las preguntas abiertas fueron analizadas cualitativamente y se refieren a los temas relevantes que dieran cuenta del fenómeno subjetivo en estudio.

El proceso de recolección de datos comenzó una vez obtenida la autorización del proyecto por la dirección del hospital y la aprobación del comité ético científico de dicho establecimiento. La recolección de los datos finalizó una vez que las categorías se saturaron, esto es, que no existieron nuevos datos importantes.

\footnotetext{
${ }^{2}$ La expresión "saturación teórica" se refiere a que en la investigación no emergieron nuevos datos importantes en una categoría. La categoría se desarrolló en términos de sus propiedades y dimensiones. Las relaciones entre las categorías se consideraron bien establecidas y validadas. La saturación teórica es de gran importancia, ya que una vez que las categorías estén bien saturadas la teoría se desarrollará de manera fluida y precisa.
}

El análisis de los datos utilizó técnicas de estadística descriptiva para presentar las características demográficas del grupo de estudio. Posteriormente se realizó el análisis cualitativo a través de teoría fundamentada, en la que se desarrollaron los procesos correspondientes a esta metodología.

En la fase inicial se procedió al escrutinio cuidadoso de los datos a través del microanálisis, durante este proceso se descubrieron conceptos y relaciones novedosas y se construyeron de manera sistémica las categorías en términos de sus propiedades y dimensiones. El proceso contempló, a continuación, una codificación abierta para identificar conceptos que representaran los fenómenos y que dieran paso a categorías; una codificación axial, relacionando las categorías con sus subcategorías, con el fin de formular explicaciones más precisas y completas de los fenómenos y, por último, una codificación selectiva, integrando y refinando las categorías para construir teoría a partir de los mismos datos.

Se realizaron 21 entrevistas, 9 correspondientes a profesionales médicos y 12 a profesionales de enfermería.

Las relaciones entre las categorías se consideraron bien establecidas y validadas.

Se confeccionaron dos matrices, cada una correspondiente a las distintas percepciones de los dos grupos de profesionales entrevistados. Para la definición de la categoría central se crearon siete categorías con sus correspondientes subcategorías, que dieron cuenta de la percepción general del ejercicio clínico de la limitación del esfuerzo terapéutico. Para estos fines, las categorías fueron orientadas, durante el transcurso del análisis, a describir el escenario a través de la percepción de los sujetos involucrados en el fenómeno en estudio: las características de los actores involucrados en el fenómeno, definiciones de conceptos fundamentales en relación con la toma de decisiones de limitación, el fundamento, los obstáculos visualizados, el proceso en el ejercicio clínico y las percepciones posteriores a la aplicación de esta práctica.

El proceso de análisis contempló la triangulación, por medio de la comparación de la inter- 
pretación de los datos de la autora con la de otro investigador con conocimientos en investigación cualitativa y teoría fundamentada, para verificar la identificación de las categorías con sus propiedades, dimensiones y el contenido homogéneo de ellas.

No hubo diferencias intercodificadores significativas sobre la elaboración y coherencia de las categorías y subcategorías. Hubo coherencia interna en relación con la selección de los datos al interior de cada categoría.

\section{Resultados}

Caracteristicas demográficas y de formación de los sujetos de estudio

Como indican las tablas 1 y 2, el $100 \%$ de los médicos posee formación de posgrado; solo 3 de los 12 profesionales de enfermería poseen formación en diplomados. Existe una alta tasa de recambio de los profesionales; los profesionales de enfermería tienen mayor antigüedad laboral. Cabe destacar que en ninguno de los dos grupos hay profesionales con formación en bioética.

Tabla 1. Datos demográficos de médicos entrevistados (9)

\begin{tabular}{|l|l|}
\hline Promedio de edad & 34 años \\
\hline Sexo & $\begin{array}{l}\text { 56\% corresponden a sexo } \\
\text { masculino } \\
44 \% \text { corresponden a sexo } \\
\text { femenino }\end{array}$ \\
\hline $\begin{array}{l}\text { Formación de } \\
\text { posgrado }\end{array}$ & $\begin{array}{l}\text { 100\% con formación de } \\
\text { posgrado en medicina } \\
\text { interna. } \\
\text { 33\% con formación en } \\
\text { subespecialidades (3 con } \\
\text { formación en subespeciali- } \\
\text { dades) }\end{array}$ \\
\hline $\begin{array}{l}\text { Formación en } \\
\text { bioética }\end{array}$ & Sin formación en bioética \\
\hline $\begin{array}{l}\text { Promedio de ejer- } \\
\text { cicio profesional } \\
\text { en unidades de } \\
\text { pacientes criticos }\end{array}$ & 5,4 años \\
\hline
\end{tabular}

Tabla 2. Datos demográficos de profesionales de enfermería entrevistadas (12)

\begin{tabular}{|l|l|}
\hline Promedio de edad & 36 años \\
\hline Sexo & $\begin{array}{l}\text { 8\% corresponde a sexo } \\
\text { masculino } \\
92 \% \text { corresponden a sexo } \\
\text { femenino }\end{array}$ \\
\hline $\begin{array}{l}\text { Formación en } \\
\text { bioética }\end{array}$ & Sin formación en bioética \\
\hline $\begin{array}{l}\text { Formación de } \\
\text { posgrado }\end{array}$ & Sin formación de posgrado \\
\hline $\begin{array}{l}\text { Especializaciones } \\
\text { mados }\end{array}$ & 3 con formación en diplo- \\
\hline $\begin{array}{l}\text { Promedio de } \\
\text { ejercicio profesio- } \\
\text { nal en unidades de } \\
\text { pacientes criticos }\end{array}$ & 8 años \\
\hline
\end{tabular}

\section{Percepciones subjetivas}

La limitación del esfuerzo terapéutico es una práctica habitual que deben enfrentar los profesionales médicos y de enfermería que laboran en unidades de pacientes críticos del hospital donde se realizó el estudio. Este hecho fue corroborado también por los sujetos de investigación. La percepción de esta experiencia presenta ciertos rasgos comunes entre médicos y enfermeros. Sin embargo, el fenómeno también exhibe ciertas particularidades propias que parecen asociarse al ejercicio profesional.

\section{Escenario clínico}

Todos los profesionales entrevistados, en general, tienen claro el perfil de los pacientes que deben atender y reconocen los criterios que deben cumplir al momento de ingresar a estas unidades. El perfil corresponde a pacientes graves, que cursan con riesgo vital y poseen posibilidades de recuperación. Los criterios corresponden a gravedad, recuperabilidad, requerimiento de técnicas de soporte vital y dependencia de la atención de enfermería.

"A la UCI llegan los pacientes más graves en el fondo del hospital" EM5. 
"Los criterios de ingreso están un poco establecidos o guiados por el grado de recuperación o las probabilidades de recuperarse que tenga el paciente" EM2.

\section{"Dependientes de la atención de enfermería" EE83.}

Los criterios expresados provienen de percepciones autónomas basadas en su práctica valorada desde la experiencia. Sin embargo, concuerdan en la mayoría de las ocasiones con los establecidos en los protocolos nacionales.

\section{Definición del concepto}

Las percepciones recogidas dan cuenta de correctas definiciones del concepto de "limitación del esfuerzo terapéutico", lo que básicamente se traduce en establecer un límite de tratamientos extraordinarios en aquellos pacientes en que se tiene la certeza que no existen posibilidades de recuperación. El concepto se asocia además a condiciones fundamentales que deben ir de la mano a la hora de ejercer esta práctica, y es aquí donde comienzan a visualizarse las primeras dimensiones éticas del problema: "Es tratar de darle quizá a veces un confort o un alivio... o sea... no es cortar todo, eso es lo que yo entiendo por lo menos, no abandonarlo" EM1. Como se observa en la cita anterior, está presente una dimensión emocional que acompaña a la reflexión centrada en la importancia que se le otorga a no abandonar a los pacientes limitados y otorgarles un buen morir: "Limitar el esfuerzo es tratar de ayudar a la gente al bien morir, y el bien morir es eso, no seguir haciendo injuria en seguir atentando contra la vida de una persona, si en el fondo es una paciente que ya no tiene posibilidades de que su salud mejore" EE1.

\section{Emergencia de problemas éticos}

En el caso de los profesionales médicos, la emergencia de problemas éticos en la práctica clínica está vinculada con cuatro puntos específicos: la instauración o retiro de tratamientos, la dimensión emocional, la confusión del concepto y la evolución de los pacientes que se encuentran hospitalizados en unidades críticas.

Para los profesionales es más difícil retirar un tratamiento que ya se instauró, asunto que no tiene

\footnotetext{
${ }^{3}$ EE: Entrevista enfermera; EM: Entrevista médico. El número es correlativo en relación al orden en que se realizaron las entrevistas.
}

que ver con la indicación, sino con el problema que surge en relación con decidir quién es el indicado para ejecutar esta acción. En la mayoría de las ocasiones los profesionales tienen claro, desde el punto de vista técnico, que lo que procede es una limitación, pero las emociones juegan un papel fundamental, ya que la ejecución de esta práctica les deja una sensación de dejar morir al paciente. Para ellos, el concepto se puede confundir principalmente con el de eutanasia, reconociéndose cierta asociación entre ambos conceptos: "Temor o mito de que uno vaya a cometer cierta eutanasia, como que uno haga una intervención que acelere el proceso de la muerte, como que uno quiere tal vez que se produzca el proceso en forma natural, pero cuesta un poco cuando se ha realizado una intervención descalar de dicha intervención" EM2.

Para los profesionales de enfermería, los problemas éticos se originan fundamentalmente por la falta de toma de decisiones de los profesionales médicos y el desacuerdo que existe entre los equipos tratantes. Estos profesionales reconocen que la práctica de la limitación debe ser ejercida en aquellos pacientes que poseen el criterio de irrecuperabilidad, pero en ocasiones los profesionales médicos escapan un poco de estos criterios y extreman medidas con el fin de derrotar la muerte, cuando lo que deberían hacer es darle paso y dejar que el proceso ocurra en las mejores condiciones posibles. La retirada de los tratamientos crea mayor dificultad, se percibe como una situación muy complicada, pero principalmente porque para los médicos es difícil reconocer cuándo parar con los tratamientos instalados: "Ellos deberían ser más criteriosos y tener conciencia de que, después de cierta edad, si no hay más posibilidades terapéuticas en un paciente, por qué incurrir en esa injuria y contra la vida de un paciente, cuando no tiene el sentido que debería tener" EE1.

Existe desconocimiento en relación con las implicancias éticas. Estos profesionales reconocen ciertas técnicas como parte de la limitación, sin serlo.

\section{Fundamentos para la toma de decisiones}

Uno de los fundamentos principales, reconocido por ambos grupos de estudio al momento de enfrentarse a esta toma de decisiones, es la postura que tenga la familia. 
Se torna fundamental que estas decisiones sean conversadas y consentidas por los familiares. En general declaran que si fue así no existen problemas y el éxito se basa fundamentalmente en la buena relación clínica.

En ambos grupos de estudio se identifican aspectos técnicos fundamentales en el momento de tomar decisiones de limitación: factores clínicos, claridad del diagnóstico, patologías asociadas y la calidad de vida posterior. El criterio médico y la edad del paciente se agregan como criterios no absolutos para fundamentar las decisiones.

Los profesionales de enfermería reconocen que la decisión sobre limitación del esfuerzo terapéutico la toma el médico y consideran que su opinión no es tomada en cuenta; en las situaciones en las que se ven involucrados es a causa de que ellos preguntan. Existe una minoría de percepciones en las que los profesionales reconocen su inclusión, pero cabe destacar que es solo en aquellos casos de profesionales que poseen mayor antigüedad laboral.

\section{Obstáculos en la toma de decisiones}

Para los profesionales médicos, si bien en el momento de aplicar la limitación del esfuerzo terapéutico las decisiones se toman fundamentalmente con el apoyo familiar, paradojalmente la familia se identifica como el principal obstáculo, y por dos razones: porque las familias no comprenden la situación y esto básicamente por la mala comunicación que existe entre ellos y el equipo; y porque, a raíz de esta oposición familiar, los profesionales se posicionan temerosos frente a un posible proceso de judicialización.

En relación al papel profesional, se detecta como principal obstáculo el desacuerdo entre los equipos profesionales y la diversidad de opiniones frente a una determinada situación.

Entre los obstáculos reconocidos por los profesionales de enfermería nace solo una subcategoría en la que existe uniformidad de percepciones. Para ellos el papel que cumplen los profesionales médicos es el principal obstáculo, porque la falta de experiencia los lleva a una demora en la toma de decisiones, a lo que se suma el reconocimiento de cierto temor a la judicialización, lo que dificulta aún más la decisión.

\section{Ejercicio de la limitación del esfuerzo terapéutico}

Una vez ejercida la limitación, las percepciones concuerdan en relación con las técnicas limitadas. Éstas corresponden a técnicas de soporte vital, tales como intubación endotraqueal, ventilación mecánica invasiva, aporte de drogas vasoactivas, antibióticos de última generación; el no ingreso a unidades de mayor complejidad también es reconocido como un tipo de limitación.

Cuando la limitación del esfuerzo terapéutico se inicia, no se abandona al paciente; se da prioridad a que se encuentre junto a su familia en los momentos finales de la vida, que cuente con confort $\mathrm{y}$ alivio del sufrimiento.

Generalmente, los tratamientos ofrecidos a estos pacientes consisten en analgesia, sedación y alimentación, siempre con el fin de proporcionar un alivio; estas son percepciones concordantes al momento del análisis.

Sin duda, se rescata como tema clave proporcionar una muerte digna, y esto significa estar acompañado de la familia, sin dolor, sin sufrimiento y lo más tranquilo posible.

\section{Discusión}

La limitación del esfuerzo terapéutico es una práctica que deben enfrentar habitualmente los profesionales médicos y de enfermería que laboran en unidades de pacientes críticos del hospital donde se realizó el estudio. La percepción de esta experiencia presenta ciertos rasgos comunes entre médicos y enfermeros; sin embargo, también presenta ciertas particularidades propias que parecen asociarse al ejercicio profesional, lo que influye sobre las respectivas percepciones e informa por tanto la acción.

Las percepciones recogidas dan cuenta sobre correctas definiciones del concepto de limitación del esfuerzo terapéutico. Esta corrección se refiere a su adecuación con las definiciones oficiales sobre el concepto.

A pesar de que, en un principio, los conceptos se encuentren claros, en el momento de ejercer 
la limitación del esfuerzo terapéutico emergen dificultades relacionadas principalmente con el desconocimiento de las implicancias éticas de este tipo de decisiones al final de la vida, la mala relación clínica y la inexperiencia de los profesionales.

En relación con el desconocimiento de las implicancias éticas, la interferencia de las propias emociones, interpretado por los sujetos como un tema valórico, juega un papel fundamental. Estos resultados coinciden con lo mencionado en la literatura sobre el tema, en la que se reconoce que el tema valórico afecta las decisiones de limitación del esfuerzo terapéutico. "Se producen frecuentes conflictos relacionados con las decisiones que se toman en torno a los enfermos críticos que están en el final de su vida, especialmente con la limitación de tratamientos de soporte vital (LTSV). La mayoría son conflictos de valores entre las diversas partes implicadas: el paciente, sus familiares y/o representantes, los profesionales sanitarios y la institución"(5).

Otro problema fundamental es que, de acuerdo con la percepción de los profesionales, los problemas se ocasionan en gran medida por la falta de comunicación y de trabajo en equipo para la limitación del esfuerzo terapéutico.

Los conceptos técnicos involucrados en esta práctica se encuentran claros en ambos grupos de estudio. Sin embargo, el desconocimiento de fundamentos éticos, sumado al papel de las emociones, la mala comunicación y relación clínica al interior del equipo posicionan a las personas en un constante cuestionamiento sobre la toma de decisiones respecto de la limitación.

En relación con los aspectos técnicos utilizados después del ejercicio de la limitación, existe claridad y uniformidad en todas las percepciones. Los componentes fundamentales de la terapia son mantener medidas de tratamientos ordinarias, tales como hidratación, alimentación y analgesia.

En todo momento se visualiza como componente fundamental no abandonar al paciente y aliviar su sufrimiento, cuestión concordante con relación a los fines de la medicina.

Un lugar central en la percepción del trabajo lo ocupa la conciencia sobre el hecho de que el trabajo realizado es especial, no es cualquier trabajo. Trabajar con seres humanos hace a estos profesionales cuestionarse permanentemente sobre la importancia del respeto a la dignidad que merecen las personas. Estas consideraciones, a juicio de la investigadora, son implícitas, es decir, no parecen el producto de una reflexión consciente e intencionada. Se refleja en un continuo cuestionamiento a favor de no abandonar a los pacientes y permitir que los momentos en el final de la vida se desarrollen de la mejor forma posible, para, de esta forma, ofrecerles lo que a su juicio es una muerte digna.

El paciente que se encuentra en una condición de irrecuperabilidad y en quien se ejerció la limitación del esfuerzo terapéutico no debe ser abandonado ni olvidado. Se da prioridad a mantenerlo en un ambiente digno y de respeto, evitando la invasión, tranquilo y confortado. Ambos grupos de estudio reconocen que los componentes fundamentales de una muerte digna son que el paciente se encuentre con su familia, sin dolor, sin sufrimiento y $\sin$ angustia.

Pese a que ambos grupos de estudio perciben que los pacientes de unidades críticas son pacientes graves y de alta complejidad, los médicos consideran que estos pacientes son recuperables. En cambio para los profesionales de enfermería son pacientes con múltiples patologías y muy dependientes de la atención de enfermería, y por tanto la recuperabilidad no se plantea como un criterio absoluto a abordar. Para los médicos entrevistados, la limitación del esfuerzo terapéutico consiste principalmente en la limitación de tratamientos en aquellos pacientes que no tienen probabilidades de recuperación; para los profesionales de enfermería, en cambio, se focaliza en establecer un límite a estos tratamientos, para dar paso a un buen morir.

Como consecuencia, los problemas éticos relacionados con la limitación del esfuerzo terapéutico también presentan distintas percepciones. La instauración o retiro de tratamientos y la evolución de los pacientes parecen ser las principales fuentes de problemas éticos para los médicos. En cambio, los profesionales de enfermería reconocen que estos problemas se originan fundamentalmente por 
detalles en el proceso de toma de decisiones o desacuerdo entre los equipos tratantes.

Cuando existe una buena comunicación con la familia, no existen en general problemas para lo toma de decisiones, pero cuando la familia no comprende el escenario, la decisión se posterga hasta encontrar el acuerdo. En esto, uno de los principales factores involucrados es el temor que existe por parte de los médicos a la judicialización de la situación.

Las características técnicas en relación con los tratamientos que se limitan y los posteriores a la limitación se encuentran claras al momento del análisis.

Los profesionales de enfermería refieren en forma casi unánime su falta de inclusión en la toma de decisiones sobre limitación del esfuerzo terapéutico, con excepción de aquellos que poseen mayor antigüedad laboral.

\section{Conclusiones}

La limitación del esfuerzo terapéutico es un tema de gran interés entre los problemas éticos en el final de la vida. La propuesta de esta investigación aporta positivamente al estudio de estos problemas en las unidades críticas de nuestro país, lo que se evidencia a través de falta de estudios publicados en relación con el tema. Significa una importante apertura al estudio y discusión, con el fin de mejorar los procesos y unificar criterios en relación con esta práctica.

Este estudio logra descubrir un espacio valioso de reflexión sobre las implicancias éticas de la limitación del esfuerzo terapéutico, tanto para los sujetos de estudio como para la propia investigadora, lo que sin duda constituye un punto de partida para la experiencia de autorreflexión y del cuestionamiento de las prácticas de todos los involucrados en esta dimensión de la práctica clínica. Se pone de manifiesto la necesidad de reconsiderar los procesos de toma de decisiones en relación a la limitación del esfuerzo terapéutico y de reflexionar sobre la importancia y necesidad de ejercer estas prácticas junto a una reflexión bioética indispensable.
A raíz del imparable avance de la ciencia, se torna fundamental reflexionar de la mano de una ética de la responsabilidad: "La técnica moderna ha introducido acciones de magnitud tan diferente, con objetos y consecuencias tan novedosos, que el marco de la ética anterior no puede ya abarcarlos" (6). Para Hans Jonas, la ética no debe cambiar su esfera ni debe ser modificada en su esencia, lo que procede es originar una nueva esfera que nos sitúe en un nuevo escenario que debe ser revisado en profundidad.

En las unidades de pacientes críticos se viven situaciones al límite, originadas por la manipulación de la muerte que se logra a través de la utilización de las técnicas de soporte vital. Este estudio ha dejado de manifiesto que los principales conflictos se originan en los procesos de toma de decisiones, mediadas por el desconocimiento de las implicancias éticas y una escasa reflexión sobre el tema.

Si bien históricamente la discusión se ha generado en relación al derecho a la vida, es momento que hablemos de un derecho a la muerte, un derecho que debe poseer, indiscutiblemente como característica fundamental, el respeto a la dignidad.

Una vez que indiscutiblemente se aproxima la muerte no se debe buscar su derrota y es necesaria una responsabilidad en las acciones que contribuya a ofrecer una muerte digna y no realizar acciones que solo entorpezcan el proceso y alarguen la agonía y el sufrimiento: "Hasta la más seria de todas las tareas médicas, la evitación de la muerte prematura, puede sustituir a la naturaleza por el arte como escala de qué es "prematuro" y extender la medida natural de la finitud humana con técnicas heroicas de prolongación de la vida o retraso de la muerte. Este será uno de nuestros temas, bajo el título de responsabilidad ética" (7).

Dado que las implicancias éticas surgen como las principales dificultades percibidas por los equipos responsables de aplicar la limitación del esfuerzo terapéutico en la etapa del final de la vida, de este estudio se desprende el deber de crear instancias educativas en bioética, con el fin de unificar criterios y esclarecer los distintos escenarios en que se desarrollan los problemas éticos del final de la vida. Tal esfuerzo tendría el objetivo de contex- 
tualizar y precisar las nociones y normas básicas que permitan fortalecer la toma de decisiones, para actuar con mayor seguridad como equipo $y$, por lo tanto, beneficiar a los pacientes.

\section{Referencias}

1. Gracia D. Ética de los confines de la vida. Bogotá: El Búho: 2003.

2. Fernández R, Baigorri F, Artiagas A. Limitación del esfuerzo terapéutico en cuidados intensivos. ¿Ha cambiado en el siglo XXI? Revista de Medicina Intensiva 2005; 29(6): 338-334.

3. Strauss A, Corbin J. Bases de la investigación cualitativa. Técnicas y procedimientos para desarrollar la teoria fundamentada. Medellín: Universidad de Antioquia; 2002.

4. Horwitz Campos N. Dato y norma. El sentido de los métodos empiricos en la bioética. Santiago de Chile: Sociedad Chilena de Bioética; 2008.

5. Monzón-Marín JM, Saralegui Reta I, Abizanda i Campos R, et al. Recomendaciones de tratamiento al final de la vida del paciente crítico. Revista de Medicina Intensiva 2008; 32(3): 121-133.

6. Jonas H. El principio de responsabilidad. Barcelona: Herder; 1995.

7. Jonas H. Técnica, medicina y ética. Barcelona: Paidós; 1997.

Recibido: 5 de septiembre de 2011

Aceptado: 19 de noviembre de 2011 